\title{
Investigations of subsurface structures and buried inhomogeneities by photothermal inspection
}

\author{
by REIGL M.*, GAPP M.*, SCHMITZ B. ${ }^{*}$, STEIN J. ${ }^{\circ}$, GOCH G.*0, SEIDEL.$^{+}{ }^{+}$and \\ WALTHER H.G. ${ }^{+}$
}

* Universität Ulm, Abt. Meß-, Regel- und Mikrotechnik (MRM), Bereich Meßtechnik, Albert-Einstein-Allee 41, D-89069 Ulm, Germany

- Institut für Lasertechnologien in der Medizin an der Universität UIm (ILM), Arbeitsgruppe Lasermeßtechnik, Helmholtzstr. 12, D-89081 Ulm, Germany

+ Friedrich-Schiller-Universität Jena (FSU), Institut für Optik und Quantenelektronik, MaxWien-Platz 1, D-07743 Jena, Germany

\begin{abstract}
Among optical methods the photothermal radiometry offers a new metrological technique for the successful investigation of subsurface defects, buried inhomogeneities and structures beneath an optical opaque surface. In these investigations, the thermal waves reflect several sets of information about the inner and outer structures, about optical, thermal and mechanical properties.

The purpose of this contribution is the presentation of theoretical calculations for simulation of measuring signals and reconstruction of buried inhomogeneities from measured amplitude and phase signals. Hence, experimental results can yield a reliable defect identification and identification of surface and subsurface thermal features.
\end{abstract}

\section{Introduction}

Since over one decade, thermal waves have been used for detecting subsurface hidden structures which have dimensions of $\mathrm{mm}$ down to $\mu \mathrm{m}$ in a nondestructive manner. Theoretical works by Busse and Rosencwaig [1] started discussing photoacoustic effects in 1980 , extended to thermal waves by Mandelis [2]. The approach we follow in our work is given by an analogy to the optical projection of the structure using the point spread function $[3,4]$. Our special point of interest is focused on the measurement of material properties and the detection of defects by photothermal microscopy with radiometry and optical beam deflection $[5,6,7]$. Moreover, not only measurements and their simulations are carried out, but also the reverse process is taken into account. The starting point is the inversion of the point spread function. So, depth, shape and thermal parameters of the defect can be determined. Other works, carried out e.g. by Crowther et. al. [8], use the inverse scattering algorithm with the Green's function when measuring pulse echoes or thermal tomography with back propagation in the wave field approach by Padé et. al. [9].

\section{Theory of photothermal imaging}

\subsection{Photothermal imaging of thermal inhomogeneities}

According to the theory of Walther et. al. [3,4] subsurface structures and buried Inhomogeneities, inspected by a modulated continuous wave heat source, are imaged to the measuring signal in analogy to an optical projection by a transfer function, called the point spread function (psf). The projection $S$ is given by the convolution of the defect distribution $W$ (in the depth $z_{0}$ ) and the psf $G$ :

$$
S(x, y)=W(x, y) * G(x, y)
$$


http://dx.doi.org/10.21611/qirt.1994.035

The psf is a function of thermal parameters and the modulation frequency $\omega$ :

$$
G(x, y)=\frac{\pi}{2} \sigma^{2} \frac{1}{\sqrt{x^{2}+y^{2}+z_{0}^{2}}} e^{-\sigma\left(z_{0}+\sqrt{x^{2}+y^{2}+z_{0}^{2}}\right)}\left[\frac{A z_{0}}{\sqrt{x^{2}+y^{2}+z_{0}^{2}}}\left(1+\frac{1}{\sigma \sqrt{x^{2}+y^{2}+z_{0}^{2}}}\right)+B\right](2)
$$

where $A=\Delta \mathcal{\kappa} / \mathcal{\kappa} \quad$ difference of therm. conductivity between defect and base material

$B=\Delta(\rho c) /(\rho c)$ difference of the specific heat capacity

$\sigma=(1+i) / \mu=(1+i) Y$

$\mu=\sqrt{2 \kappa / \rho C \omega}$ thermal diffusion length

$Y \quad$ frequency parameter

The detection of hidden structures is done by scanning the surface in two directions, so the above functions are described in these two coordinates. The measured signal consists of an amplitude and a phase shift relative to the modulation of the excitation source. They are reflected by the real part and the imaginary part of $S$ and are called the amplitude and phase contrast, respectively. These contrast functions depend on the modulation frequency; they are illustrated in figure 1.

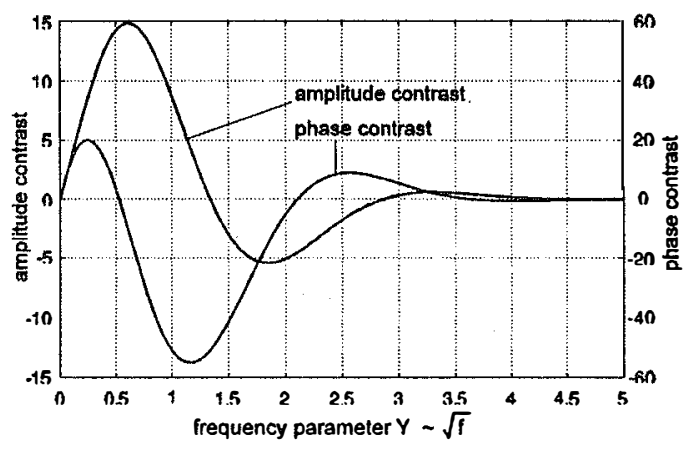

Fig. 1: Amplitude and phase contrast as a function of the modulation frequency

\subsection{Reconstruction of a buried structure}

For a given signal $S(x, y)$ (either measured or simulated) the original defect distribution $W(x, y)$ can be calculated by a deconvolution of the signal and the psf $G(x, y)$. Simulations were carried out varying the parameters of the psf in order to estimate the premissable deviation of the unknown parameters from the real ones [10].

The simulations show that the depth of the defect may deviate by approx. a factor 2

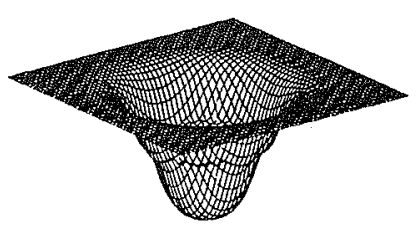

a)

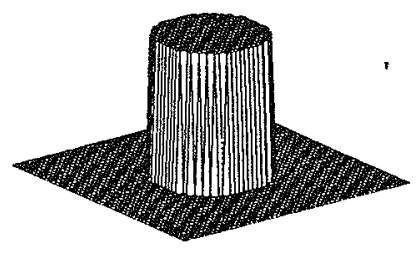

b)

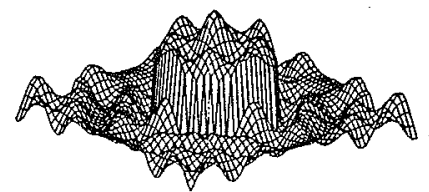

c)

Fig. 2a-2c: Reconstructed defect of a disc shaped defect: a) simulated measuring signal, b) reconstructed defect with correct parameters, c) reconstructed defect with wrong set of parameters

and the defect strengthens in the order of $15 \%$ from the real parameters. Figure $2 a$ illustrates the simulated phase contrast of a given disc shaped defect. Depending on the depth, the defect either appears spread or, in a very small depth, almost identical with the original defect distribution. 
http://dx.doi.org/10.21611/qirt.1994.035

If the defect is now calculated by the deconvolution on the basis of the signal, the resulting defect distribution for known parameters of the psf is - as expected - the given disc shaped defect, which is shown in figure $\mathbf{2 b}$. But normally the parameters of the psf (depth of the defect, defect strengthens) are unknown. Therefore, it is necessary to estimate and to optimize these parameters. The defect strengthens are easier to estimate, because the basis material is known and only the material of the inhomogeneities have to be estimated. The depth of the defect can be estimated by varying this parameter by the defect reconstruction. This optimization is carried out by minimizing the mean value of the reconstructed defect.

If, for example, the depth within the psf in the defect calculation is varied, the reconstructed defect, as illustrated in figure $2 c$, is difficult to detect, especially if the signal is noisy.

\section{Experimental configuration}

For the experimental verification of the model-based calculations we prepared several well-defined samples and carried out investigations under different conditions.

The experimental setup is shown in figure 3. Herein, the detection schemes of both the photothermal radiometry and the optical beam deflection are realized. The thermal waves are excited by a modulated argon laser. The beam is tightly focused on the sample's surface within a spot size of about $10 \mu \mathrm{m}$. In photothermal radiometry (PTR) the variations of the surface temperature are monitored by a JTcooled MCT-detector. When measuring the optical beam deflection (OBD) the local thermal response of the periodically heated solid is detected by the deflection of a probe laser beam while it crosses the air region above the heated surface. The deflection is caused by the temperature dependent variations of the refraction index in this small air volume. Both the amplitude and the phase signals were imaged (simultaneously the radiometric and OBD-signals) reconstructing the subsurface features. The samples themselves are mounted on computer controlled $x$ - $y$-stages for moving them relative to the detectors and the laser beams.

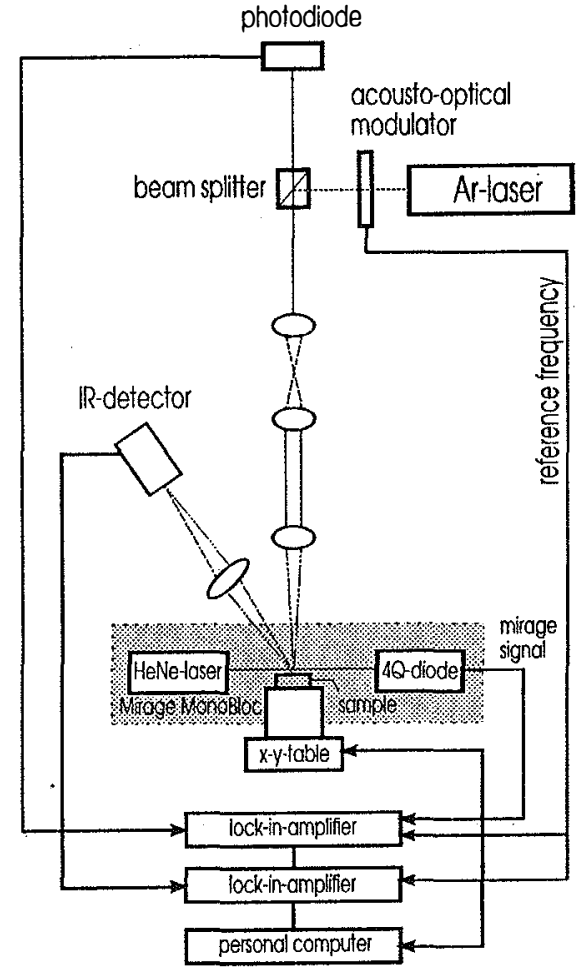

Fig. 3: Configuration for photothermal experiments

\section{Experimental results and reconstructions}

\subsection{Sample 1: Glass ball in tin}

As the first sample to be presented, a glass ball (diameter appr. $1 \mathrm{~mm}$ ) is embedded in tin. The whole sample is covered with a thin layer of tin $(\approx 100 \mu \mathrm{m})$ and polished. Thus, the 'thermal inhomogeneity' in the sample remains completely invisible from the outer surface. The sample has been scanned at several modulation frequencies. The 'defect' 
http://dx.doi.org/10.21611/qirt.1994.035

could be clearly resolved in the amplitude and phase signals up to about $10 \mathrm{kHz}$. The approach of the psf has been applied to the experimental results. The contrasts at two different places (as indicated in figure 4-c) of the phase angle of the defect signal compared with the phase signal of the undisturbed area are shown in figure 4-d. Obviously, there are some indications that the contrast depends on the defect depths. The qualitative conformity between the theoretical predictions and the experimental results illustrate the relevance of the considered imaging model.

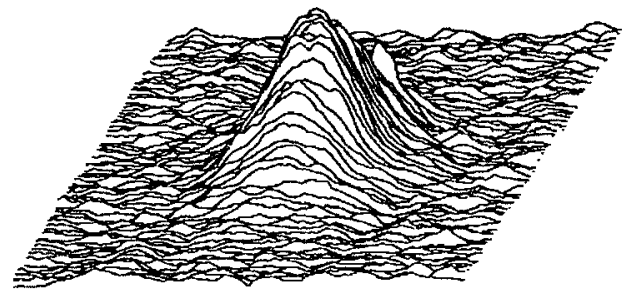

a)

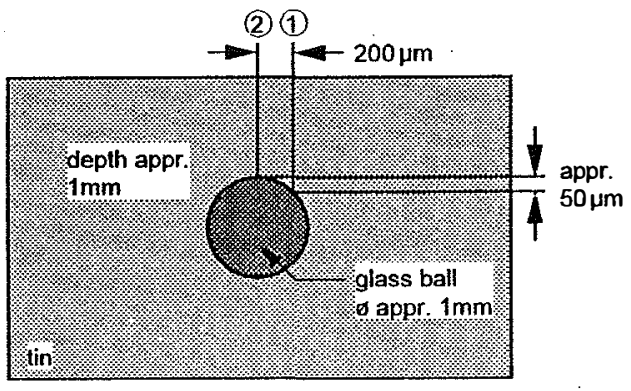

c)

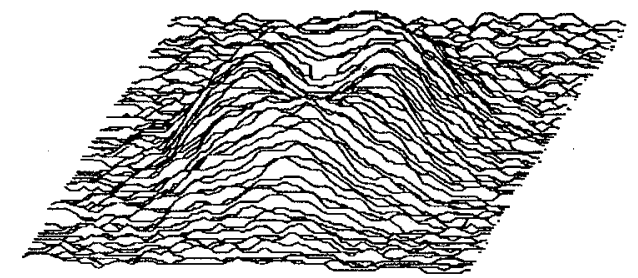

b)

Phase contrast (in deg.)

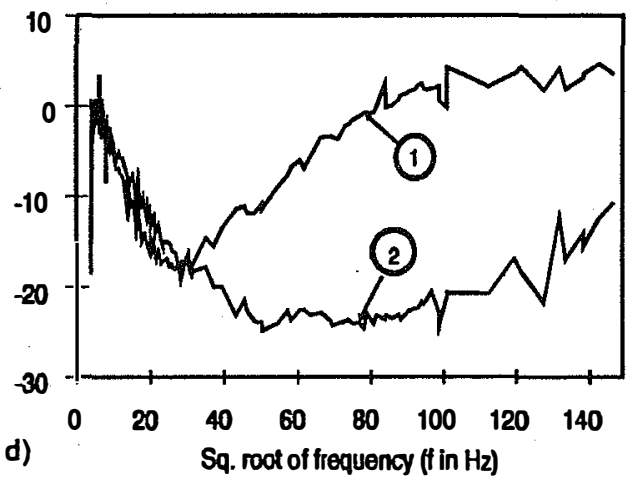

Fig. 4a-4d: Glass ball in tin: scanned images: a) amplitude image, b) phase image, scanned area: 1.5 - $1.2 \mathrm{~mm}$; c) design of the sample; d) contrasts at two measured points above the defect

\subsection{Sample 2: Gold disc in copper}

A gold disc (as shown in figure 5) with a diameter of $800 \mu \mathrm{m}$ on a copper substrate was covered by a galvanic deposited layer of copper with a thickness of about 10-30 $\mu \mathrm{m}$. The disc itself had a thickness of $2 \mu \mathrm{m}$. The sample was scanned over the whole area and inspected both by the IR-detector and by the optical beam deflection.

The measured signal in figure $6 a$ reflects the disc shape with a camber of the edge las a result of the production process). The inner area of the measuring signal shows a raise compared to the surrounding

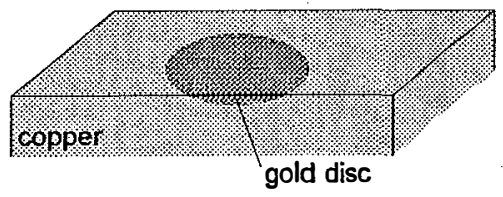

Fig.5: Gold disc in copper substrate region indicating the slightly different thermal parameters of the gold disc. The reconstructed defect geometry in figure $6 b$ shows a great similarity to the measuring signal, but the important parameter determined from the reconstruction is not directly 
http://dx.doi.org/10.21611/qirt.1994.035

monitored in this figure: the obtained defect depth varies from 10-30 $\mu \mathrm{m}$ which corresponds to the specification of the manufacturer.

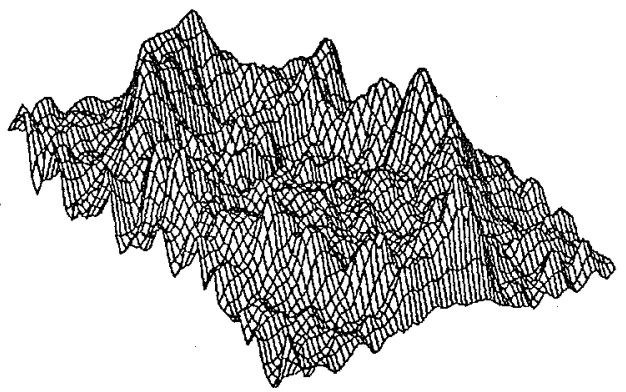

a)

Fig. 6a-6b: Gold disc: a) scanned image (phase image); b) reconstructed defect with a determined depth of 10-30 $\mathrm{\mu m}$

\subsection{Sample 3: Copper wire in tin}

A copper wire with a diameter of $70 \mu \mathrm{m}$ was placed diagonally in a tin body (figure 7). The surface was ground until the wire was visible, so the wire passes the surface with an elliptic shape. An area including the wire at the surface and the wire vanishing in the depth was inspected.

The measured signal is illustrated in figure $8 a$ and

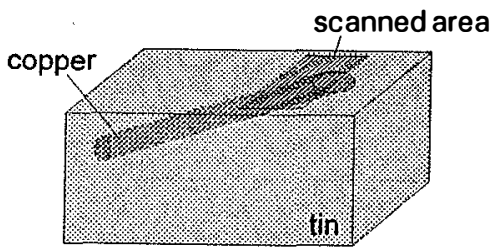

Fig. 7: Copper wire in tin consists of two parts: the wire passing through the surface (right part of the signal showing the immersion); the interesting part is in the left area displaying a raised area which reflects the wire vanishing in the depth, decreasing signal with increasing depth of the wire (from right to left). In the reconstructed defect geometry in figure $8 b$ the increasing depth of the wire is indicated by the increasing values in the left part; the camber on the right side results from the wire to be seen at the surface.

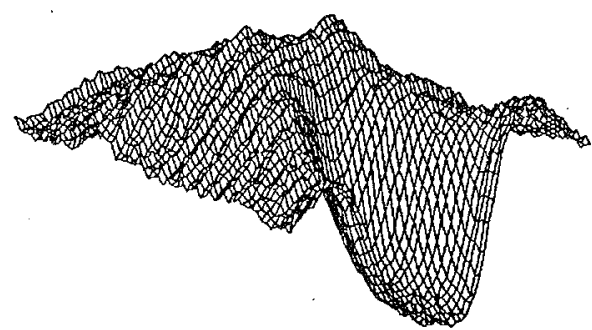

a)

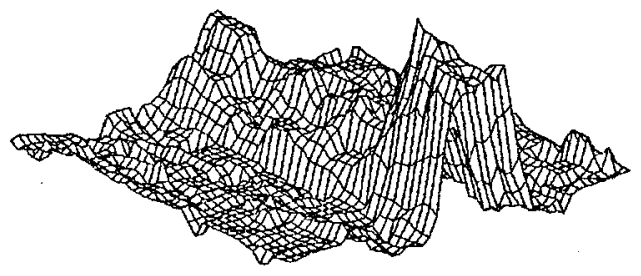

b)

Fig. 8a-8b: Copper wire: a) scanned image (phase image); b) reconstructed defect

\section{Conclusions}

In this paper we presented after a brief survey of the theory of the point spread function a procedure for the reconstruction of a measured defect distribution and its parameters like 
http://dx.doi.org/10.21611/qirt.1994.035

e.g. the depth of the defect. The theoretical predictions like frequency dependence and determinability of defect depth and shape have been confirmed by several measurements.

\section{Acknowledgments}

The authors wish to thank Michael Reick for his support in measuring the samples and we also thank Dr. A. Zielonka from the "Forschungsinstitut für Edelmetalle und Metallchemie" in Schwäbisch Gmünd for the preparation of the gold disc sample.

This work is supported by a grant of the federal state of Baden-Württemberg, Germany Nr. II 7532.294-4/1.

\section{REFERENCES}

[1] BUSSE (G.) ROSENCWAIG (A.): Subsurface Imaging with Photoacoustics, Appl. Phys. Lett. 36 (1980), p. 815

[2] MANDELIS (A.) : Theory of Photothermal-Wave Diffraction and Interference in Condensed Media, J. Opt. Soc. Am., A6 (1989), p. 298-308

[3] FRIEDRICH (K.), HAUPT (K.), SEIDEL (U.), WALTHER (H.G.): Definition, Resolution and Contrast in Photothermal Imaging, J. Appl. Phys. 72, 8 (1992), p. $3759-3764$

[4] SEIDEL (U.), HAUPT (K.), WALTHER (H.G.), BURT (J.), BEIN (B.K.): Analysis of the Detectability of Buried Inhomogeneities by Means of Photothermal Microscopy, J. Appl. Phys. 75, 9 (1994), p. 4396-4401

[5] GOCH (G.), SCHMITZ (B.), REICK (M.): Photothermal Sensing Techniques for Measuring Material Properties and Near-Surface Defects, Annals of the CIRP 42, 1 (1993), p.623-626

[6] GOCH (G.), SEIDEL (U.), SCHMITZ (B.) , WALTHER (H.G.): Applications of Photothermal Measurement Techniques for the Detection of Subsurface Defects and Thermal Inhomogeneities, XIII IMEKO World Congress, Turin, Sept. 1994, to be published [7] SEIDEL (U.), HAUPT (K.) SCHMITZ (B.), GOCH (B. G.) WALTHER (H. G.): Principles and Applications of Photothermal Microscopy, XIII IMEKO World Congress, Turin, Sept. 1994, to be published

[8] CROWTHER (D.J.), FAVRO (L. D.) KUO (P.K.), R.L. Thomas: Inverse scattering algorithm applied to infrared thermal wave images, J. Appl. Phys. 74, 9 (1993), p. 58285834

[9] PADÉ (O.), MANDELIS (A.): Computational thermal-wave slice tomography with backpropagation and transmission reconstructions, Rev.Sci.Instrum. 64,12 (1993),p.35483562

[10 REIGL (M.)ET AL.: Reconstruction of a Subsurface Structure From Photothermal Data, to be published 